\title{
Decametric AGNs: FIRST and NVSS Maps and Radio Spectra
}

\author{
O. V. Verkhodanov, N. V. Verkhodanova \\ Special Astrophysical Observatory, Nizhnij Arkhyz, Russia, 369167 \\ H. Andernach \\ Depto. de Astronomía, Apdo. Postal 144, Univ. Guanajuato, Mexico
}

\begin{abstract}
Radio sources from the decametric UTR-2 catalog were crossidentified with other radio catalogs at higher frequencies. We used the CATS database to extract all sources within the UTR beam size $\left(\sim 40^{\prime}\right)$ to find candidate radio identifications. Using the least squares method, we fitted the spectrum of each source with one of a set of curves. We extracted NVSS and FIRST radio images for the radio-identified sources, and looked for a possible relation between size and spectral index.
\end{abstract}

A radio survey obtained with the UTR telescope (Kharkov, Ukraine) at frequencies $10-25 \mathrm{MHz}$ has resulted in a catalog of 1822 sources (Braude et al. 1978-1994; www.ira.kharkov.ua/UTR2). Covering about $30 \%$ of the sky north of $-13^{\circ}$ declination, this survey is presently the lowest-frequency source catalog of its size, and thus provides an ideal basis to study the little known optical identification content of sources selected at decametric frequencies. The rather large uncertainties of UTR positions $\left(\sim 0.7^{\circ}\right)$ require an iterative process for finding radio counterparts at successively higher frequencies (and thus higher positional accuracy). In this we were aided by selecting previously cataloged sources from the CATS database (Verkhodanov et al. 1997) in a box of RA $\times D E C=40^{\prime} \times 40^{\prime}$ centred on the nominal UTR position. The "raw" spectra given by these fluxes were refined using computer charts of source locations around UTR positions. All counterparts from TXS, GB6 and PMN within circles of $1^{\prime}$ radius were considered one source. Groups of sources lying further apart were assigned separate spectra, each with the UTR flux as an upper limit.

We were able to fit spectra for all but 7 of the 2314 radio counterparts to UTR sources. Fits were either straight $(\mathrm{S})$, convex $\left(\mathrm{C}^{-}\right)$, or concave $\left(\mathrm{C}^{+}\right)$ curves in the $\lg \nu-\lg S$ plot. The distribution of radio source spectra among the various spectral types is given in Table 1 . The resulting catalog (Verkhodanov et al. 2000) includes information from a large number of electronically available catalogs of radio, infrared, optical and X-ray sources.

The majority of UTR sources (97\%) have an identification with NVSS objects (Condon et al., 1998) (a total of 2253 IDs), and all UTR objects with $\delta>30^{\circ}$ (1143 objects) have IDs in FIRST (White et al., 1997). 552 sources were resolved into components either in the FIRST ( $5^{\prime \prime}$ beam) or NVSS (15" beam) catalogs within a circle of $60^{\prime \prime}$. 
Table 1. Distribution of radio continuum spectral types of 2307 radio counterparts to UTR sources, where $X=\log _{10}$ (frequency/MHz), and $\mathrm{Y}=\log _{10}$ (flux density in Jy)

\begin{tabular}{llrr}
\hline Spectral class & Fitting function & $\mathrm{N}$ & $\%$ \\
\hline Straight (S) & $Y=+A+B * X$ & 894 & 39 \\
Convex $\left(C^{+}\right)$ & $Y=+A \pm B * X-C * X^{2}$ & 184 & 8 \\
Concave $\left(C^{-}\right)$ & $Y=+A-B * X+C * X^{2}$ & 1150 & 50 \\
or & $Y= \pm A \pm B * X+C * E X P(-X)$ & 79 & 3 \\
\hline \hline
\end{tabular}

Table 2. Flux and size distribution of UTR counterparts in FIRST and NVSS: median value for various ranges of spectral index. $N$ is the number of objects.

\begin{tabular}{ccccccc}
\hline Sp. index & \multicolumn{3}{c}{ median FIRST } & \multicolumn{3}{c}{ median NVSS } \\
range & $N$ & flux (mJy) & size $\left({ }^{\prime \prime}\right)$ & $N$ & flux (mJy) & size $\left({ }^{\prime \prime}\right)$ \\
\hline$-0.9 \div-1.0$ & 88 & 514 & 26 & 183 & 574 & 24 \\
$-1.0 \div-1.1$ & 93 & 363 & 19 & 168 & 365 & 22 \\
$<-1.1$ & 75 & 193 & 12 & 185 & 212 & 17 \\
\hline \hline
\end{tabular}

We extracted NVSS and VLA maps of sources with steep and straight power law spectra, and grouped them according to their spectral slopes: (1) $-0.9>\alpha>-1.0,(2)-1.0>\alpha>-1.1$ and (3) $\alpha<-1.1\left(S \propto \nu^{\alpha}\right)$. NVSS objects were taken outside of the Galactic plane $\left(|b|>15^{\circ}\right)$, while all the FIRST sources have $|b|>20^{\circ}$. 536 NVSS objects and 256 FIRST objects were selected. Parameters of source samples are provided in Table 2. Surprisingly, the median source size decreases with steepening radio spectrum. However, the dispersion in size is so large that there is no significant correlation between spectral index and source size.

\section{References}

Braude S.Ya. et al. 1978,1979,1981,1985,1994, Ap\&SS, 54,37; 64,73; 74,409; 111,$1 ; 213,1$

Condon J.J., Cotton W.D., Greisen E.W., Yin Q.F., et al. 1998, AJ 115, 1693 Verkhodanov O.V., Trushkin S.A., Andernach H., \& Chernenkov V.N. 1997, in "Astronomical Data Analysis Software and Systems VI", eds. G.Hunt \& H.E.Payne, ASP Conf. Ser. 125, 322 (astro-ph/9610262)

Verkhodanov O.V., Andernach H., Verkhodanova N.V. 2000, Bull. Spec. Astroph. Obs. 49, 53 (astro-ph/0008431)

White R.L., Becker R.H., Helfand D.J., \& Gregg M.D. 1997, ApJ 475, 479 